\title{
Small curvature laminations in hyperbolic 3-manifolds
}

\author{
WILLIAM BRESLIN
}

\begin{abstract}
We show that if $\mathcal{L}$ is a codimension-one lamination in a finite volume hyperbolic 3 -manifold such that the principal curvatures of each leaf of $\mathcal{L}$ are all in the interval $(-\delta, \delta)$ for a fixed $\delta \in[0,1)$ and no complementary region of $\mathcal{L}$ is an interval bundle over a surface, then each boundary leaf of $\mathcal{L}$ has a nontrivial fundamental group. We also prove existence of a fixed constant $\delta_{0}>0$ such that if $\mathcal{L}$ is a codimension-one lamination in a finite volume hyperbolic 3 -manifold such that the principal curvatures of each leaf of $\mathcal{L}$ are all in the interval $\left(-\delta_{0}, \delta_{0}\right)$ and no complementary region of $\mathcal{L}$ is an interval bundle over a surface, then each boundary leaf of $\mathcal{L}$ has a noncyclic fundamental group.
\end{abstract}

$57 \mathrm{M} 50$

\section{Introduction}

In [9], Zeghib proved that any totally geodesic codimension-one lamination in a closed hyperbolic 3-manifold is a finite union of disjoint closed surfaces. In this paper we investigate whether a similar result holds for codimension-one laminations with small principal curvatures. We will prove the following theorems:

Theorem 1 Let $\delta \in[0,1)$. If $\mathcal{L}$ is a codimension-one lamination in a finite volume hyperbolic 3-manifold such that the principal curvatures of each leaf of $\mathcal{L}$ are $\mathrm{ev}$ erywhere in $(-\delta, \delta)$ for a fixed constant $\delta \in[0,1)$ and no complementary region of $\mathcal{L}$ is an interval bundle over a surface, then each boundary leaf of $\mathcal{L}$ has a nontrivial fundamental group.

Theorem 2 There exists a fixed constant $\delta_{0}>0$ such that if $\mathcal{L}$ is a codimension-one lamination in a finite volume hyperbolic 3-manifold such that the principal curvatures of each leaf of $\mathcal{L}$ are everywhere in $\left(-\delta_{0}, \delta_{0}\right)$ and no complementary region is an interval bundle over a surface, then each boundary leaf of $\mathcal{L}$ has a noncyclic fundamental group. 


\section{Examples}

Let $\mathcal{L}$ be a codimension-one lamination in a complete hyperbolic 3-manifold $M$. Let $L$ be a leaf of $\mathcal{L}$ and endow it with the path metric induced from $M$. Let $\widetilde{L}$ be the universal cover of $L$ and lift the inclusion $i_{L}: L \rightarrow M$ to a map $\tilde{i_{L}}: \widetilde{L} \rightarrow \mathbb{H}^{3}$. A map $f: X \rightarrow Y$ from a metric space $X$ to a metric space $Y$ is a $(k, c)$-quasi-isometry if $\frac{1}{k} d_{X}(a, b)-c \leq d_{Y}(f(a), f(b)) \leq k d_{X}(a, b)+c$. The leaf $L$ is quasi-isometric if the map $\tilde{i}_{L}$ is a $(k, c)$-quasi-isometry for some $k, c$. The lamination $\mathcal{L}$ is quasi-isometric if each leaf of $\mathcal{L}$ is quasi-isometric for the same fixed constants $k, c$.

Let $\delta \in(0,1)$. If the principal curvatures of $\tilde{i}_{L}(\widetilde{L})$ are everywhere in $(-\delta, \delta)$, then the map $\tilde{i}_{L}$ is a $(k, c)$-quasi-isometry for constants $k, c$ depending only on $\delta$ (see Thurston [8]. Also see Leininger [6] for an elementary proof).

The constant $\delta_{0}$ in Theorem 2 is less than 1, so a lamination satisfying the hypotheses of Theorem 1 or Theorem 2 is necessarily quasi-isometric. Thus it makes sense to ask whether these results hold for general quasi-isometric laminations.

Quasi-isometric laminations with no compact leaves Cannon and Thurston [3] proved that the stable and unstable laminations of the suspension of a pseudo-Anosov homeomorphism of a closed surface are quasi-isometric, and each leaf is a plane or annulus in this case. In addition to these examples, Fenley [5] produced infinitely many examples of closed hyperbolic 3-manifolds with quasi-isometric laminations in which each leaf is an annulus, a mobius band, or a plane. Note that Theorem 2 implies that the examples of Cannon-Thurston and Fenley cannot have principal curvatures everywhere in the interval $\left(-\delta_{0}, \delta_{0}\right)$.

One can also ask if we need to require that no complementary region is an interval bundle over a surface.

Small curvature laminations with simply connected boundary leaves Let $S$ be a closed totally geodesic embedded surface in a closed hyperbolic 3-manifold $M$. Let $N(S)=S \times[0,1]$ be a closed embedded neighborhood of $S$ in $M$. If the neighborhood $N(S)$ is small then the surfaces $S \times t$ will have small principal curvatures. Since $\pi_{1}(S)$ is left-orderable, there exist faithful representations $\rho: \pi_{1}(S) \rightarrow$ Homeo( $\left.[0,1]\right)$ such that some points have trivial stabilizers (see Calegari [2]) The foliated bundle whose holonomy is $\rho$ has a leaf which is simply connected. Replace $N(S)$ with this foliated bundle. We can blow up the simply connected leaf and remove the interior to get a lamination which is $C^{\infty}$ close to the original (so that the leaves have small principal curvatures) and such that some boundary leaf is simply connected. See Calegari [1] to see why the foliated bundle can be embedded in $M$ so that the leaves are smooth. Note that this lamination has a complementary region which is an interval bundle over a surface. 
Small curvature laminations with no compact leaves One may also construct small curvature laminations in closed hyperbolic 3-manifolds with no compact leaves. The author would like to thank Chris Leininger for describing the following construction. The idea is to construct a small curvature branched surface in a closed hyperbolic 3 -manifold which has an irrational point in the space of projective classes of measured laminations carried by the branched surface. A lamination corresponding to this irrational point will contain no compact leaves. There are totally geodesic immersed closed surfaces in the figure-eight knot complement $M_{8}$ arbitrarily close to any plane in the tangent bundle (see Reid [7]). Using this and the fact that $\pi_{1}\left(M_{8}\right)$ is LERF, one can find two such surfaces which lift to embedded surfaces $S_{1}$ and $S_{2}$ in a finite cover $M$ of $M_{8}$ which intersect in a nonseparating (in both surfaces) simple closed geodesic $l$ at an arbitrarily small angle. Flatten out the intersection to get a branched surface with small principal curvatures in which $S_{1}$ connects one side of $S_{2}$ to the other side. The branched surface has three branch sectors (an annulus, $S_{1} \backslash l$, and $S_{2} \backslash l$ ) and one branch equation $\left(x_{1}=x_{2}+x_{3}\right)$. A solution to the branch equation in which two coordinates are not rationally related (eg, $\left.x_{1}=1 / 2, x_{2}=1 / \pi, x_{3}=1 / 2-1 / \pi\right)$ will correspond to a lamination with no compact leaves which can be isotoped to have small principal curvatures. Since the leaves do not have any cusps, we can fill the cusps of $M$ to get a small curvature lamination in a closed hyperbolic 3-manifold with no compact leaves.

\section{Proof of Theorem 1}

Let $\epsilon>0$ be so small that if $P_{1}, P_{2}, P_{3}$ are three disjoint smoothly embedded planes in hyperbolic 3 -space with principal curvatures in $(-1,1)$ which intersect the same $\epsilon$-ball, then one of the $P_{i}$ separates the other two.

Let $\mathcal{L}$ be a codimension-one lamination in a finite volume hyperbolic 3-manifold $M$ such that the principal curvatures of each leaf are everywhere in the interval $(-\delta, \delta)$ for some $\delta \in(0,1)$. Assume that no complementary region of $\mathcal{L}$ is an interval bundle over a surface. Let $\tilde{\mathcal{L}}$ be the lift of $\mathcal{L}$ to $\mathbb{H}^{3}$. Since every leaf of $\mathcal{L}$ has principal curvatures everywhere in $(-\delta, \delta)$, the lamination $\mathcal{L}$ is a quasi-isometric lamination, and cannot be a foliation of $M$ by Fenley [4].

Let $L_{0}$ be a boundary leaf of $\mathcal{L}$. Suppose, for contradiction, that $\pi_{1}\left(L_{0}\right)$ is trivial, which implies that $L_{0}$ has infinite area. Since $M$ is closed, $L_{0}$ must intersect some fixed compact ball in $M$ infinitely many times. Thus given any integer $k$, we can find a point $y_{k}$ in $L_{0}$ such that the next leaf over on the boundary side of $L_{0}$ is within $1 / k$ of $y_{k}$. 
Let $\widetilde{L}_{0}$ be a lift of $L_{0}$ to $\mathbb{H}^{3}$. Lift the points $y_{k}$ to a fixed fundamental domain of $\widetilde{L}_{0}$ and call them $y_{k}$. Let $\widetilde{L}_{k}$ be the next leaf over from $\widetilde{L}_{0}$ which is within $1 / k$ of $y_{k}$. We now have a sequence of leaves $\widetilde{L}_{k}$ in $\widetilde{\mathcal{L}}$ on the boundary side of $\widetilde{L}_{0}$ such that for each $k$ the distance from $\tilde{L}_{k}$ to $y_{k}$ is less than $1 / k$, and there is no leaf of $\mathcal{L}$ between $\widetilde{L}_{0}$ and $\widetilde{L}_{k}$. We also have that $\partial \widetilde{L}_{0} \neq \partial \widetilde{L}_{k}$ for all $k$, because otherwise the region between $L_{0}$ and $L_{k}$ would be an interval bundle in the complement of $\mathcal{L}$.

Let $k$ be so large that $1 / k<\epsilon / 8$. Since $\tilde{L}_{k}$ eventually diverges from $\tilde{L}_{0}$ we can find a point $x_{k} \in \widetilde{L}_{0}$ such that the distance from $x_{k}$ to $\tilde{L}_{k}$ is exactly $\epsilon / 8$. Let $b_{k}$ be the $(\epsilon / 32)$-ball tangent to $\widetilde{L}_{0}$ at $x_{k}$ on the boundary side of $\widetilde{L}_{0}$.

We will show that infinitely many of the balls $b_{k}$ are disjointly embedded in $M$, contradicting the fact that $M$ has finite volume. Suppose that $\gamma\left(b_{l}\right) \cap b_{k} \neq \varnothing$ for some integers $l, k$ and some $\gamma$ in $\pi_{1}(M)$. Note that $\gamma\left(\widetilde{L}_{0}\right) \neq \widetilde{L}_{0}$, since $L_{0}$ has trivial fundamental group. Now $\widetilde{L}_{0}, \widetilde{L}_{k}$, and $\gamma\left(\widetilde{L}_{0}\right)$ all intersect some $\epsilon$-ball, so we must have that one of them separates the other two. Since there are no leaves of $\widetilde{\mathcal{L}}$ between $\widetilde{L}_{0}$ and $\widetilde{L}_{k}$, and $\gamma\left(\widetilde{L}_{0}\right)$ is closer to $x_{k}$ than $\widetilde{L}_{k}$, we must have that $\widetilde{L}_{0}$ separates $\widetilde{L}_{k}$ and $\gamma\left(\widetilde{L}_{0}\right)$ (see Figure $1\left(\right.$ a)). Also note that $\widetilde{L}_{0}, \widetilde{L}_{k}$, and $\gamma\left(\widetilde{L}_{l}\right)$ are all on the boundary side of $\gamma\left(\tilde{L}_{0}\right)$ (ie, the side which contains the ball $\gamma\left(b_{l}\right)$ ).

Now we will show no matter where $\gamma$ sends $\widetilde{L}_{l}$, we get a contradiction. We cannot have $\gamma\left(\widetilde{L}_{l}\right)=\widetilde{L}_{k}$, because this would imply that $\gamma^{-1}\left(\widetilde{L}_{0}\right)$ separates $\widetilde{L}_{l}$ and $\tilde{L}_{0}$. Thus we have $\gamma\left(\widetilde{L}_{l}\right) \neq \tilde{L}_{k}$.

Since $\tilde{L}_{0}, \tilde{L}_{k}$, and $\gamma\left(\tilde{L}_{l}\right)$ all intersect some fixed $\epsilon$-ball, we must have that one of them separates the other two. We cannot have that $\gamma\left(\tilde{L}_{l}\right)$ separates $\widetilde{L}_{0}$ and $\tilde{L}_{k}$, because there are no leaves of $\tilde{\mathcal{L}}$ between $\widetilde{L}_{0}$ and $\widetilde{L}_{k}$ (See Figure $1(\mathrm{~b})$ ). If $\widetilde{L}_{0}$ separates $\widetilde{L}_{k}$ and $\gamma\left(\tilde{L}_{l}\right)$, then $\gamma\left(\widetilde{L}_{l}\right)$ is between $\widetilde{L}_{0}$ and $\gamma\left(\widetilde{L}_{0}\right)$, so that $d\left(x_{l}, \widetilde{L}_{l}\right)=$ $d\left(\gamma\left(x_{l}\right), \gamma\left(\widetilde{L}_{l}\right)\right) \leq \epsilon / 16$ which is a contradiction (see Figure $1(\mathrm{c})$ ). Thus $\widetilde{L}_{0}$ cannot separate $\widetilde{L}_{k}$ and $\gamma\left(\tilde{L}_{l}\right)$. If $\tilde{L}_{k}$ separates $\tilde{L}_{0}$ and $\gamma\left(\tilde{L}_{l}\right)$, then $\gamma^{-1}\left(\tilde{L}_{k}\right)$ separates $\widetilde{L}_{0}$ and $\widetilde{L}_{l}$ which is a contradiction (see Figure $1(\mathrm{~d})$ ). Thus $\widetilde{L}_{k}$ cannot separate $\widetilde{L}_{0}$ and $\gamma\left(\tilde{L}_{l}\right)$. We have shown that $\tilde{L}_{l}$ has nowhere to go under the map $\gamma$, so that $\gamma\left(b_{l}\right) \cap \gamma\left(b_{k}\right)=\varnothing$ for any integers $l, k$ and any $\gamma \in \pi_{1}(M)$. This implies that $M$ contains infinitely many disjoint $(\epsilon / 32)$-balls, contradicting the fact that $M$ has finite volume.

\section{Proof of Theorem 2}

Let $\epsilon>0$ be so small that if $P_{1}, P_{2}, P_{3}$ are three disjoint smoothly embedded planes in hyperbolic 3 -space with principal curvatures in $(-1,1)$ which intersect the same 


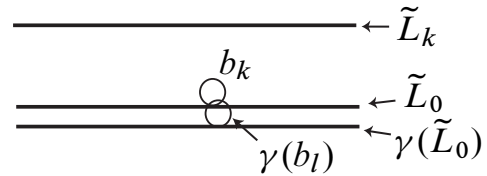

(a)

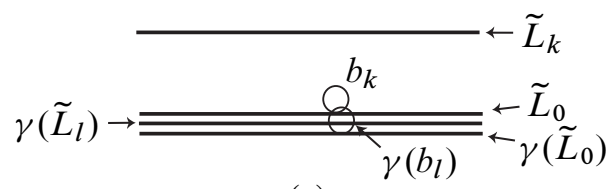

(c)

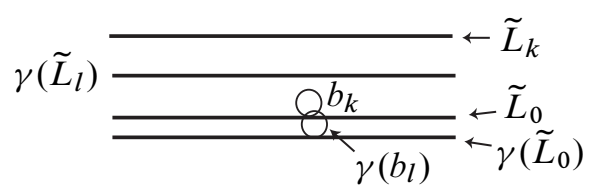

(b)

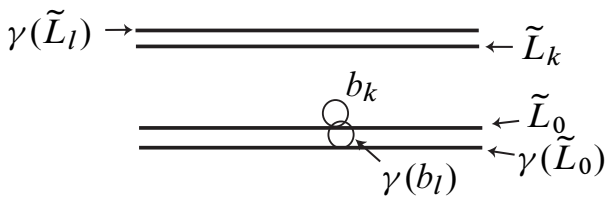

(d)

Figure 1: (a) $\tilde{L}_{0}$ separates $\widetilde{L}_{k}$ and $\gamma\left(\widetilde{L}_{0}\right)$. (b) $\gamma\left(\widetilde{L}_{l}\right)$ cannot separate $\tilde{L}_{0}$ and $\widetilde{L}_{k}$. (c) $\widetilde{L}_{0}$ cannot separate $\widetilde{L}_{k}$ and $\gamma\left(\widetilde{L}_{l}\right)$. (d) $\widetilde{L}_{k}$ cannot separate $\widetilde{L}_{0}$ and $\gamma\left(\tilde{L}_{l}\right)$.

$\epsilon$-ball, then one of the $P_{i}$ separates the other two. Let $\delta_{0}>0$ be so small that if a smooth curve $\gamma:(-\infty, \infty) \rightarrow \mathbb{H}^{3}$ in $\mathbb{H}^{3}$ with endpoints in $\partial \mathbb{H}^{3}$ has curvature at most $\delta_{0}$ at each point, then $\gamma(t)$ is in the $(\epsilon / 2)$-neighborhood of the geodesic of $\mathbb{H}^{3}$ with the same endpoints.

Let $\mathcal{L}$ be a codimension-one lamination in a finite volume hyperbolic 3-manifold $M$ such that the principal curvatures of each leaf are everywhere in the interval $\left(-\delta_{0}, \delta_{0}\right)$. Assume that no complementary region of $\mathcal{L}$ is an interval bundle over a surface. Let $\widetilde{\mathcal{L}}$ be the lift of $\mathcal{L}$ to $\mathbb{H}^{3}$. As in the proof of Theorem $1, \mathcal{L}$ cannot be a foliation. Let $L_{0}$ be a boundary leaf of $\mathcal{L}$. Suppose, for contradiction, that $\pi_{1}\left(L_{0}\right)$ is cyclic, which implies that $L_{0}$ has infinite area. Since $M$ is closed, $L_{0}$ must intersect some fixed compact ball in $M$ infinitely many times. Also, by Theorem 1 , we know that $\pi_{1}\left(L_{0}\right)$ is nontrivial, so that $\pi_{1}\left(L_{0}\right) \approx \mathbb{Z}$.

Let $\widetilde{L}_{0}$ be a lift of $L_{0}$ to $\mathbb{H}^{3}$. Since $L_{0}$ intersects a fixed compact ball in $M$ infinitely many times, we can find a sequence of points $y_{k}$ in $\widetilde{L}_{0}$ such that the closest leaf of $\widetilde{\mathcal{L}}$ to $y_{k}$ on the boundary side of $\widetilde{L}_{0}$ is within $1 / k$ of $y_{k}$. Let $\widetilde{L}_{k}$ be the leaf which is closest to $y_{k}$ on the boundary side of $\widetilde{L}_{0}$. Note that there is no leaf of $\widetilde{\mathcal{L}}$ between $\widetilde{L}_{0}$ and $\widetilde{L}_{k}$. We have $\partial \widetilde{L}_{0} \neq \partial \widetilde{L}_{k}$ for all $k$, because the complement of $\mathcal{L}$ contains no interval bundle components. We may assume that all $y_{k}$ are contained in a fixed fundamental domain $\mathcal{D}$ of $\widetilde{L}_{0}$, and that $y_{k}$ converge to a point $y_{\infty} \in \partial \widetilde{L}_{0}$.

For $k$ large enough we have $\partial \widetilde{L}_{0} \neq \partial \tilde{L}_{k}$ and $d\left(y_{k}, \tilde{L}_{k}\right) \leq \epsilon / 8$, so that we can find a point $x_{k}$ such that $d\left(x_{k}, \tilde{L}_{k}\right)=\epsilon / 8$. 
Case 1 We can choose the sequence of points $x_{k} \in \widetilde{L}_{0}$ to be contained in a fixed fundamental domain $D$ of $\widetilde{L}_{0}$ such that $x_{k}$ exit an end of $D$ whose projection to $M$ has infinite area.

Let $b_{k}$ be the $(\epsilon / 32)$-ball tangent to $\tilde{L}_{0}$ at $x_{k}$ on the boundary side of $\tilde{L_{0}}$. For $k$ large enough, say all $k$, the generator of $\operatorname{stab}_{\pi_{1}(M)}\left(\widetilde{L}_{0}\right)$ moves the center of $b_{k}$ a distance of at least $\epsilon$. Thus we can assume that $\gamma\left(b_{l}\right) \cap b_{k}=\varnothing$ for any integers $l, k$ and any $\gamma \in \operatorname{stab}_{\pi_{1}(M)}\left(\widetilde{L}_{0}\right)$.

We may now proceed as in the proof of Theorem 1 to show that $\gamma\left(b_{l}\right) \cap b_{k}=\varnothing$ for any integers $l, k$ and any $\gamma \in \pi_{1}(M)$. This again contradicts the fact that $M$ has finite volume.

Case 2 We cannot choose the sequence of points $x_{k}$ as in Case 1.

If infinitely many of the leaves $\widetilde{L}_{k}$ were distinct, then we would be able to find a sequence of points as described in Case 1 . Thus $\widetilde{L}_{k}=\widetilde{L}_{+}$for some fixed leaf $\widetilde{L}_{+} \in \tilde{\mathcal{L}}$.

Let $U$ be the component of the complement in $\partial \widetilde{L}_{0}$ of the fixed point(s) of the generator of $\operatorname{stab}_{\pi_{1}(M)}\left(\tilde{L}_{0}\right)$ which contains the point $y_{\infty}$. We will now show that $\partial \widetilde{L}_{+} \cap \partial \widetilde{L}_{0}$ must contain $U$.

Suppose that $\partial \widetilde{L}_{+} \cap \partial \widetilde{L}_{0}$ does not contain $U$. Since $d\left(y_{k}, \tilde{L}_{+}\right)<1 / k$ and $\partial \widetilde{L}_{+} \cap \partial \widetilde{L}_{0}$ does not contain $U$, we can find a sequence of points $x_{k}$ in $\widetilde{L}_{0}$ which converge to a point $x_{\infty} \in U$ with $d\left(x_{k}, \tilde{L}_{+}\right)=\epsilon / 8$. Since the point $x_{\infty}$ cannot be a fixed point of the generator of $\operatorname{stab}_{\pi_{1}(M)}\left(\widetilde{L}_{0}\right)$, a tail of the sequence $x_{k}$ must be contained in a fixed fundamental domain of $\widetilde{L_{0}}$. This contradicts the fact that we are in Case 2. Thus $\partial \widetilde{L}_{+} \cap \partial \widetilde{L}_{0}$ must contain $U$, hence must contain the fixed point(s) of the generator of $\operatorname{stab}_{\pi_{1}(M)}\left(\tilde{L}_{0}\right)$.

If the generator of $\operatorname{stab}_{\pi_{1}(M)}\left(\widetilde{L}_{0}\right)$ is parabolic, then it has only one fixed point. This implies that $\partial \widetilde{L}_{+}=\partial \widetilde{L}_{0}$, giving us a contradiction.

If the generator of $\operatorname{stab}_{\pi_{1}(M)}\left(\widetilde{L}_{0}\right)$ is loxodromic, then we can argue as above to find a leaf $\partial \widetilde{L}_{-}$of $\widetilde{\mathcal{L}}$ which contains the other component of complement in $\partial \widetilde{L}_{0}$ of the fixed points of the generator of $\operatorname{stab}_{\pi_{1}(M)}\left(\widetilde{L}_{0}\right)$. So $\partial \widetilde{L}_{+}$and $\partial \widetilde{L}_{-}$both contain the endpoints of the axis of the generator of $\operatorname{stab}_{\pi_{1}(M)}\left(\widetilde{L}_{0}\right)$. Since the principal curvatures of $\widetilde{L}_{0}, \widetilde{L}_{+}$, and $\widetilde{L}_{-}$are all in the interval $\left(-\delta_{0}, \delta_{0}\right)$, and $\partial \widetilde{L}_{0}, \partial \widetilde{L}_{+}, \partial \widetilde{L}_{-}$all contain the endpoints of the axis of the generator of $\operatorname{stab}_{\pi_{1}(M)}$, we must have that $\widetilde{L}_{0}, \widetilde{L}_{+}$, and $\tilde{L}_{-}$all intersect some fixed $\epsilon$-ball. Thus one of the three separates the other two. This gives us a contradiction since $\widetilde{L}_{+}$and $\widetilde{L}_{-}$are on the same side of $\widetilde{L}_{0}$ (ie, the boundary side) and there are no leaves of $\mathcal{L}$ between $\widetilde{L}_{0}$ and $\widetilde{L}_{+}$or between $\widetilde{L}_{0}$ and $\widetilde{L}_{-}$. 
Acknowledgements This work was partially supported by the NSF grants DMS0135345 and DMS-0602191.

\section{References}

[1] D Calegari, Leafwise smoothing laminations, Algebr. Geom. Topol. 1 (2001) 579-585 MR1875608

[2] D Calegari, Circular groups, planar groups, and the Euler class, from: "Proceedings of the Casson Fest", (C Gordon, Y Rieck, editors), Geom. Topol. Monogr. 7 (2004) 431-491 MR2172491

[3] J W Cannon, W P Thurston, Group invariant Peano curves, Geom. Topol. 11 (2007) 1315-1355 MR2326947

[4] S R Fenley, Quasi-isometric foliations, Topology 31 (1992) 667-676 MR1174265

[5] S R Fenley, Foliations with good geometry, J. Amer. Math. Soc. 12 (1999) 619-676 MR1674739

[6] C J Leininger, Small curvature surfaces in hyperbolic 3-manifolds, J. Knot Theory Ramifications 15 (2006) 379-411 MR2217503

[7] A W Reid, Totally geodesic surfaces in hyperbolic 3-manifolds, Proc. Edinburgh Math. Soc. (2) 34 (1991) 77-88 MR1093177

[8] W P Thurston, The geometry and topology of three-manifolds, Princeton Univ. Math. Dept. Lecture Notes (1979) Available at http://msri.org/publications/books/ gt3m/

[9] A Zeghib, Laminations et hypersurfaces géodésiques des variétés hyperboliques, Ann. Sci. École Norm. Sup. (4) 24 (1991) 171-188 MR1097690

Department of Mathematics, University of Michigan 530 Church Street, Ann Arbor 48109-1043, United States

breslin@umich.edu http://www $\cdot$ math.lsa $\cdot$ umich.edu/people/facultyDetail $\cdot$ php?uniqname=breslin Received: 9 February 2009 Revised: 6 March 2009 